\title{
Effect of dissolved air content on single bubble sonoluminescence
}

\author{
VIJAY H ARAKERI \\ Department of Mechanical Engineering,Indian Institute of Science,Bangalore 560012, India
}

MS received 2 January 1993

\begin{abstract}
It has been recently demonstrated that a single gas bubble in a liquid medium can be driven hard enough by an acoustic pressure field to make it emit light which is visible to the naked eye in a dark room. This phenomenon termed as single bubble sonoluminescence has shown some extraordinary physical properties. In the present investigation we have shown that dissolved air content has a significant influence on this phenomenon.
\end{abstract}

Keywords. Sonoluminescence; bubble dynamics; gas content.

\section{PACS Nos $78 \cdot 60 ; 43 \cdot 25 ; 42 \cdot 50$}

\section{Introduction}

Gaitan [1] and Gaitan and Crum [2] were the first ones to demonstrate that a single gas bubble in a liquid can be made to emit light by acoustic excitation. The light comes in repetitive bursts which is synchronous with the driving frequency [3]. Barber and Putterman [4] and Hiller et al [5] have shown some extraordinary physical features of single bubble sonoluminescence (SL). They not only showed that SL is synchronous with the frequency of the sound field but they also conservatively estimated the duration of the light flashes to 'be less than $100 \mathrm{ps}$. In addition, they found that this intense SL consists of greater than $10^{5}$ photons per burst and this can increase by a factor of up to 10 by cooling the liquid sample resulting in peak powers of as high as $30 \mathrm{mw}$. The spectrum of this bright SL has been shown to be broadband with intensity of emitted light increasing as the ultraviolet cut off of water is reached [5]. The blackbody fit to the spectrum indicates a bubble temperature of 25000 " $\mathrm{K}$ in room temperature water; the estimated bubble temperatures being in excess of $50000^{\circ} \mathrm{K}$ under cooled conditions. This synchronous picosecond sonoluminescence as ternied by Barber and Putterman [4] and Hiller et al [5] involves concentration of ambient energy by more than 12 orders of magnitude. In view of the above it is worthwhile to investigate further the parameters which control single bubble or synchronous picosecond SL. Here we have investigated the SL intensity as influenced by the dissolved gas content in the liquid sample. We have found that the SL intensity increases by more than a factor of 10 when dissolved air concentration in the liquid is reduced from about $75 \%$ to $16 \%$ of saturation.

The experimental technique of establishing stable SL is fully described in Gaitan et al [3]. Our set up is similar to the one used by 'Barber and Putterman [4]. A spherical glass flask of about $500 \mathrm{cc}$ capacity is filled with water of different dissolved air concentrations. A hollow cylindrical piezoelectric drive is epoxied to the bottom of the flask and the transducer is driven by an amplified signal from a sine generator. 


\section{Vijay H Arakeri}

A small 'pill' transducer epoxied on the flask wall and a hydrophone placed a few centimeters from the flask is used to monitor the pressure field in the flask. A photomultiplier tube (RCA 4526) with a S-20 response is used to measure SLintensity. To improve light collection, a portion of the flask opposite to the PMT is silvered. The PMT output is monitored with a digital storage oscilloscope (Tekhind 2230). The dissolved air content is measured with a Van Slyke apparatus of MERL design [6].

The temperature of the water sample was $22^{\circ} \mathrm{C}$ and the measured saturation gas concentration was 12.2 pprn. The drive frequency was about $30 \mathrm{kHz}$ and the pressure amplitudes required to establish stable SL are similar to the ones reported by Barber and Putterman [4]. Due to the short duration of the emitted light bursts it is not possible to capture a single burst from the PMT output but an average of several bursts results in a curve as shown in figure 1. This is similar to the one obtained by Barber and Putterman using a conventional PMT (they also used a micro channel PMT for better time resolution). Note that the rise time indicated by the trace is close to the stated rise time of the PMT which is about $2 \mathrm{~ns}$. It was established that $\left|V_{\max }\right|$ obtained from the trace of the type shown in figure 1 is directly related to the quantum of light output. This was done by comparing present trends of $\left|V_{\max }\right|$ with sample cooling and increasing the drive pressure amplitude with those of number of photons per burst reported by Barber and Putterman [4] and Hiller et al [5]. Thus, relative changes in light intensity were obtained by measuring $\left|V_{\max }\right|$.

The dependence of $\left|V_{\max }\right|$ with $\alpha$, the dissolved air concentration is shown in figure 2. Two different samples seem to show similar results. There is substantial increase in the SL intensity with decrease in air content. This fact could be independently verified by visual observations, since as indicated earlier, bright SL of the present type is visible to the naked eye In the dark room. It should be noted that beyond the range of a covered in figure 2, it was difficult to establish a stable light emitting bubble. Since recent findings of Hiller et al [5] seem to suggest that SL originates from high temperatures reached within the bubble, it is important to note that numerical results of Hickling and Plesset [7] show stronger collapse with less dissolved gas concentration resulting in higher bubble temperatures and pressures. This may provide an explanation for the presently observed trends. Results in figure 2 also indicate the importance of specifying dissolved gas concentration in reporting SL intensity which has not been the case so far.

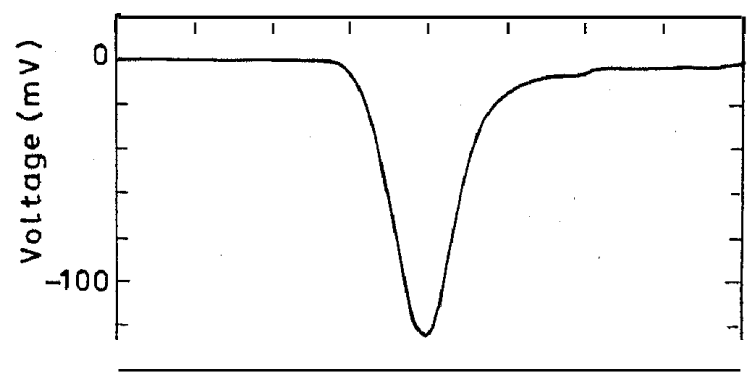

Time ( $5 \mathrm{~ns}$ per division)

Figure 1. The average of single pulse outputs of the photomultiplier tube as recorded on a digital storage oscilloscope. 


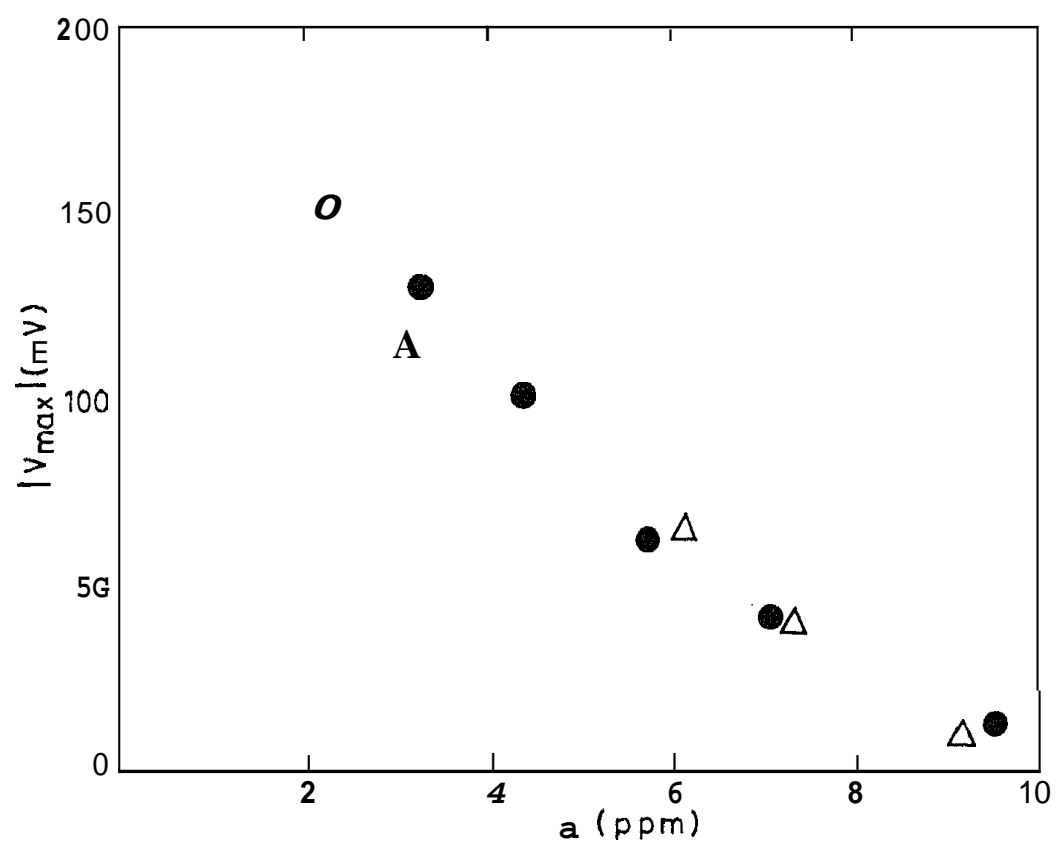

Figure 2. Effect of dissolved air concentration on sonoluminescence intensity. The two different symbols are from tests with two different samples. $T=22^{\circ} \mathrm{C}$, $\alpha_{\text {sat }}=12.2 \mathrm{ppm}$.

\section{Acknowledgement}

The author is indebted to Prof. K S Gandhi of the Chemical Engineering Department of Indian Institute of Science for bringing to his attention the publication by Barber and Putterman in Nature.

\section{References}

[1] D F Gaitan, An experimental investigation of acoustic cavitation in gaseous liquids Ph.D. Thesis Univ. of Mississippi

[2] D F Gaitan and L A Crum, J. Acoust. Soc. Am. Suppl. 87, S141 (1990)

[3] D F Gaitan, L A Crum, C C Chruch and R A Roy, J. Acoust. Soc. Am. 91, 3166 (1992)

[4] B P Barber and S J Putterman, Nature (London)352, 318 (1991)

[5] R Hiller, S J Putterman and B P Barber, Phys. Rev. Lett. 69, 1182 (1992)

[6] E E Williams, Lab. Pract. 3, 278 (1954)

[7] R Hickling and M S Plesset, Phys. Fluids 7, 7 (1964) 